\title{
A New Method of Active Power Measurement Under the Conditions of Distortion Signals Based on Multiple Wavelets
}

\author{
Zhang Xiaobing ${ }^{*}$, Li Hongguang and Cao Wei \\ Institute of Measurement, Control Technology and Communication Engineering, \\ Harbin University of Science and Technology, Harbin 150080, China \\ zhxiaobing@163.com
}

\begin{abstract}
It is discussed that a new method for active power measurement under the conditions of distortion signals. Accurate measurement of the power consumed by the distorted signals has been focused on in recent years due to its practical utilities. In this work, a mathematical description of powers is deducted in the conditions of distorted signals according to a simplified model of the electrical network. Then, a rational measuring method of the electric energy for distorted signals is proposed on the basis of the analysis of the power flow. Also, a measuring method based on multiple wavelets was employed for measuring the active power in the conditions of unsteady distorted signals. Experimental results demonstrate that, by using the simulated typical distorted signals, the proposed measurement method can produce extremely accurate results compared to theoretical results.
\end{abstract}

Keywords: active power measurement, multiple wavelets transform, mathematical model of load

\section{Introduction}

With the rapid increase of nonlinear load in power grid, the signal of grid keeps deteriorating. It has let to a complex energy exchange between grid and power load, and the reasonable electricity metering become difficult [1-2]. Nonlinear load of power grid inject distortion power into grid as a distortion source while consume electric energy, let a variety of power quality disturbance, such as voltage waveform distortion, voltage fluctuation and instantaneous pulse of PCC (Point of Common Coupling). It has been a serious problem for running of grid and sensitive electrical equipment [3-4]. So the power measurement of this nonlinear power load should be in-depth study and exploration. Scientific and reasonable method of electric energy measurement should be based on the results of detect and analyses of power flow, determine sources of pollution, individual metering distortion power and energy of inject into grid, and included in total electric energy. But the measurement method and instrument of electric energy can't be meet the needs until now. There is unreasonable that current electric energy measurement methods measure different characteristics of loads. For example, under the condition of harmonic, according to the traditional measurement method of electrical energy, the load of linear not only to pay the costs of fundamental power, but also the burden of was forced to absorb harmonics energy for its pointless and harmful, and nonlinear load on the contrary, the harmonic was pumped into the power system and pollution power grid, but the harmonics energy of inject into power system wasn't cost [5]. In effect, this mode of electric energy measurement have the function that is to encourage the user pumped harmonic into the power system, it is clear that is adverse to improve the quality of electric energy. A reasonable method is to use electric energy meter test fundamental waves, these techniques have already been experimentally applied to the non-linear loads of using electric energy measurement, which shows better results [6-7]. However, the 
fundamental wave meter is not suiTable for the electric energy measurement of the timevariable and commonly existing non-steady signals, because the harmonic model cannot reflect the real condition of grid signals. Especially the Non-stationary voltage and current signal of the nonlinear load such as impact loads, it was not described by the mathematical model of harmonic signals.

In the present situation that the signal serious distortion of power grid, it is a burning question how to accurately measure power and energy in the electric field of measuring technology and instrument. In this paper, the multiple wavelets transform been used to analysis two typical nonlinear load that semiconductor rectifiers and electric arc furnace power, and we propose the electric energy measurement method made for different band signals of different wavelet bases, and the correctness of the method was verified through simulation experiment.

\section{The Mathematical Model Of Power Grid Signals And Power Under The Conditions Of Distortion Signals}

\subsection{The Simplified Model of Power Grid Signals}

As shown in figure 1, the simplified model of power grid signals under the conditionals of distortion signals. The power supply voltage of power grid is $u(t)$ and the current is $i(t), Z_{l}$ is the impedance of circuit, $Z$ is the impedance of nonlinear load, $a$ is the energy measurement nodes of load.

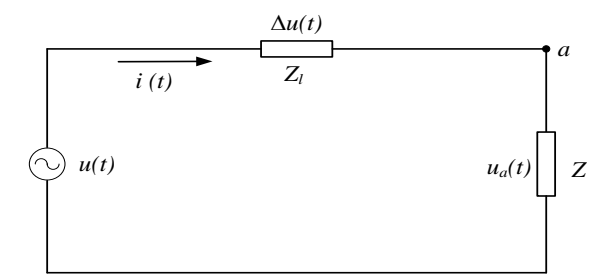

\section{Figure 1. The Electrical Network Simplified Model}

The voltage, current and its decomposition of nonlinear load are defined as follows

$$
\begin{gathered}
u_{a}(t)=u_{I}(t)+u_{s}(t) \\
i(t)=i_{I}(t)+i_{s}(t)
\end{gathered}
$$

Where $u_{I}(t)$ and $i_{I}(t)$ are the fundamental component of power signal, $u_{s}(t)$ and $i_{s}(t)$ is the distortion component of power signals.

The voltage drop of circuit impedance can be express as follow

$$
\Delta u(t)=\Delta u_{I}(t)+\Delta u_{s}(t)
$$

Where the $\Delta u_{I}(t)$ and $\Delta i_{I}(t)$ are the fundamental and distortion component of power signals of the voltage drop of circuit impedance respectively.

In the simplified model of power grid, $u_{s}(t), i_{s}(t)$ and $\Delta u_{s}(t)$ are random signals, they include not only harmonic component, also include other forms of distortion such as DC, inter-harmonic components.

\subsection{The Mathematics Model of Power Grid Signals Under The Conditions Of Distortion}

By the theory of power, we know the instantaneous power of a point

$$
p_{a}(t)=u_{a}(t) i(t)
$$


Will (1) and (2) into (3)

$$
\begin{aligned}
p_{a}(t)= & {\left[u_{I}(t)+u_{s}(t)\right]\left[i_{I}(t)+i_{s}(t)\right] } \\
& =u_{I}(t) i_{I}(t)+u_{I}(t) i_{s}(t)+u_{s}(t) i_{I}(t)+u_{s}(t) i_{s}(t) \\
& =p_{I}(t)+p_{I s}(t)+p_{s I}(t)+p_{s}(t)
\end{aligned}
$$

The average power of a point is

$$
\begin{aligned}
P_{a} & =\frac{1}{T} \int_{0}^{T} p_{a}(t) d t \\
& =\frac{1}{T} \int_{0}^{T}\left[p_{I}(t)+p_{I s}(t)+p_{s l}(t)+p_{s}(t)\right] d t \\
& =\frac{1}{T} \int_{0}^{T} p_{I}(t) d t+\frac{1}{T} \int_{0}^{T} p_{I s}(t) d t+\frac{1}{T} \int_{0}^{T} p_{s l}(t) d t+\frac{1}{T} \int_{0}^{T} p_{s}(t) d t \\
& =P_{I}+P_{I s}+P_{s l}+P_{s}
\end{aligned}
$$

Where $P_{I}, P_{I s}, P_{s I}$ and $P_{s}$ are fundamental component of power, power of by fundamental component of voltage and distortion of current, power of by distortion component of voltage and fundamental component of current and power of by distortion component of voltage and distortion component of current(here, we called distortion power) of nonlinear load.

\section{A New Method of Electrical Energy Measurement under the Conditions of Distortion}

From the result of power flow analysis and simulation, we can available that [8]:

Power of by fundamental component of voltage and current is $P_{I}>0$, it indicate that the nonlinear load absorption fundamental power from grid, so this part of electrical energy should be measurement into total energy of load consumption.

Power of by fundamental component of voltage and distortion component of current is $P_{I s}>0$, it indicate that the nonlinear load absorption distortion power from grid, so this part of electrical energy should be measurement.

Power of by distortion component of voltage and fundamental component of current is $P_{s I}<0$, it indicate that this component power is not be consumption, but as the distortion power back to the grid. However, the form of current back to the power grid is fundamental, and don't bring pollution to the power grid, so it should be measured.

Power of by distortion component of voltage and current $P_{s}>0$, it indicate that this part of power not been consumption, but as the distortion power back to the grid. However, the form of current back to the power grid is distortion, and bring pollution to the power grid, therefore not be measured.

In summary, we proposal a universal method of electrical measurement for the nonlinear load

$$
\begin{gathered}
P=P_{I}+P_{I s}+P_{s I} \\
P=P_{a}-P_{S}
\end{gathered}
$$

Where, $P_{a}$ is the measure power of measurement node $a, P_{s}$ is the distortion part of $P_{a}$.

When the $u_{s}(t)$ and $i_{s}(t)$ are the higher-order harmonics, type become on

$$
P=P_{I}+P_{I s}+P_{s I}=P_{I}
$$

According to the type, we manufacture the meter of electrical energy measurement degenerate as a fundamental watt-hour meter, so it also applies to the harmonic user. 


\section{Multiple Wavelets Transform}

\subsection{Wavelet Transform}

Using the algorithm of wavelet decomposition and reconstruction the voltage and current, we can find the component of fundamental and the component of distortion under the conditions of distortion signals.

From the MRA theory, $u(t)$ and $i(t)$ can be broken down into different frequency components

$$
\begin{aligned}
& u(t)=\sum_{k} c_{0}(k) \varphi(t-k)+\sum_{k} \sum_{j=0}^{J-1} d(k) 2^{j / 2} \psi\left(2^{j} t-k\right) \\
& i(t)=\sum_{k} c_{0}^{\prime}(k) \varphi(t-k)+\sum_{k} \sum_{j=0}^{J-1} d_{j}^{\prime}(k) 2^{j / 2} \psi\left(2^{j} t-k\right)
\end{aligned}
$$

Where $J$ is the level of decomposition $c_{j}(k)$ and $c_{j}^{\prime}(k)$ is the scale coefficient, $d_{j}(k)$ and $d_{j}^{\prime}(k)$ is the wavelet coefficient. If the scaling function is a set of orthogonal basis, then $c_{j}(k), c_{j}^{\prime}(k), d_{j}(k)$ and $d_{j}^{\prime}(k)$ can be description respectively

$$
\left.\begin{array}{l}
\left.\begin{array}{l}
c_{j}(k)=\left\langle u(t), \varphi_{j, k}(t)\right\rangle \\
d_{j}(k)=\left\langle u(t), \psi_{j, k}(t)\right\rangle
\end{array}\right\} \\
c_{j}^{\prime}(k)=\left\langle i(t), \varphi_{j, k}(t)\right\rangle \\
\left.d_{j}^{\prime}(k)=\left\langle i(t), \psi_{j, k}(t)\right\rangle\right\}
\end{array}\right\}
$$

From the two-scale function, we know

$$
\left.\begin{array}{r}
c_{j-1}(k)=\sum h(n-2 k) c_{j}(n) \\
d_{j-1}(k)=\sum_{n}^{n} g(n-2 k) c_{j}(n) \\
c_{j-1}^{\prime}(k)=\sum_{j-1}^{n} h(n-2 k) c_{j}^{\prime}(n) \\
d_{j-1}^{\prime}(k)=\sum_{n}^{n} g(n-2 k) c_{j}^{\prime}(n)
\end{array}\right\}
$$

Where $h(k)$ and $g(k)$ are called low-pass filter and high-pass filter respectively, and meet

$$
g(k)=(-1)^{k} h(1-k)
$$

The initial-scale coefficient $c_{j}(k)$ and $c_{j}^{\prime}(k)$ are acquired by directly sampling $u(t)$ and $i(t)$. If the Sample Rate of signal is greater than Nyquist frequency, then, the signal $u(t)$ and $i(t)$ have a good approximated using $c_{j}(k)$ and $c_{j}^{\prime}(k)$. In this way, from the initial scale coefficient, we can decomposition signal of voltage and current into different band layer-by-layer.

\subsection{Wavelet Transform and Optimal Wavelet Basis Select}

In order to overcome the shortcoming of single wavelet transform decomposition and reconstruction distortion signals, we combined with characteristic of all kinds of wavelet basis and fundamental component of signal and distortion component of signal, we proposed a method that using different wavelet basis decompose different distortion signal for different band.

The very important point of wavelet transform in engineering is how to select an optimal wavelet basis, because of the same problem is analyzed have different result using different wavelet basis. In this paper, for the distortion signal of the semiconducting 
rectifier and the harmonic model of electrical arc furnace, the optimal wavelet basis for the different band was select to decomposition and reconstruction the signal. The Db40 wavelet basis and the fundamental component of signal have the same form, and the Sym11 wavelet basis and the distortion component of signal have the same form, so with the $\mathrm{Db}$ and Sym series wavelet basis, we study the power measurement issue of two type nonlinear load in power grid [9].

The model of semiconductor rectifier comprised parts of harmonic and impulse function models, and the model of electrical arc furnace comprised parts of harmonic model. And the Db40 wavelet basis have a good detection effect for the sine class signal, the sym11 wavelet basis have a good detection effect for the distortion signals. So in this article, for the characteristics of sine and distortion signals, we use different wavelet basis to decomposition and reconstruction signal.

\section{Simulation Test of Electrical Energy Measurement}

In this section, we have a simulation experiment about electrical energy measurement of semiconductor rectifier and electrical arc furnace, using the Db40 and Sym11 wavelet basis decomposition the voltage and current of load and get the fundamental component and distortion component, respectively, and then compare with simple using Db40 wavelet basis according to the reasonable electrical energy measurement method measure electrical energy. Simulation experiment using Matlab7.4.0.739 in this article.

\subsection{Active Power Measurement of Semiconductor Rectifier}

It is calculated in theoretical that the active power theory value of semiconductor rectifier consumption, $\mathrm{fs}=12800 \mathrm{~Hz}$ is sampling frequency of simulation, $\mathrm{n}=1280$ is sampling points, and the signal is decomposed into 6 levels, Figure 2 and Table 1 are the result of using Db40 wavelet basis decomposition, Figure 3 and Table 2 are the distortion component result of using Db40 wavelet basis decomposition, and the fundamental component result of using Sym11 wavelet basis decomposition,
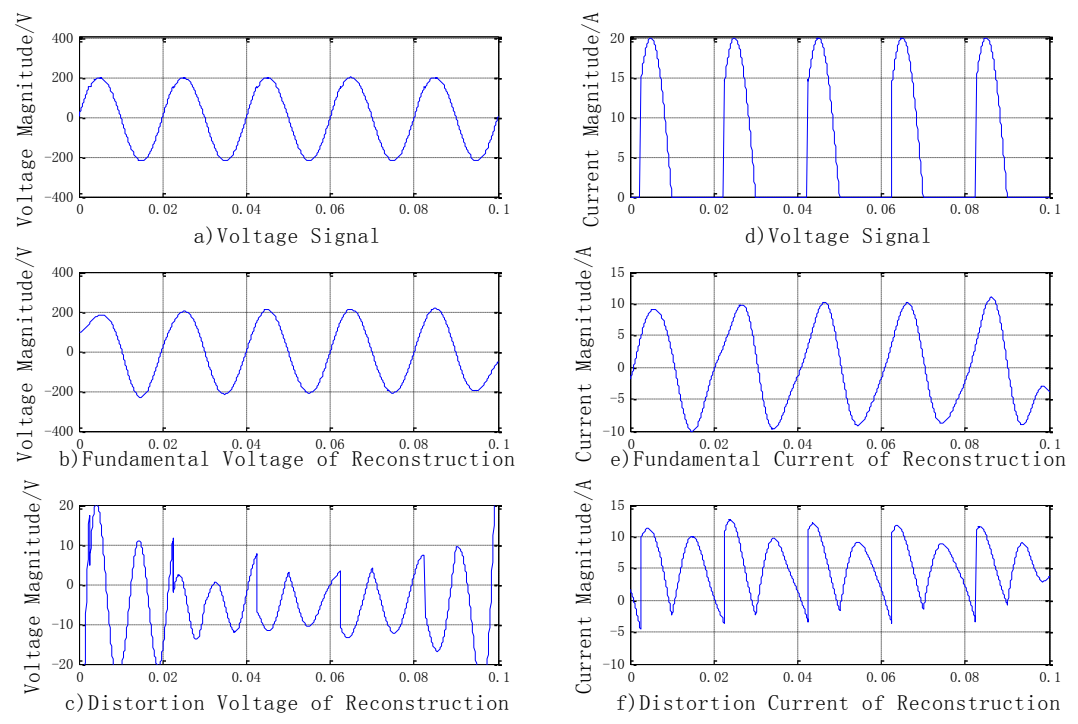

\section{Figure 2. Distortion Wave and Fundamental Wave of Current and Voltage by Using Db40 Wavelets Decomposed}


Table 1. Results of Power Measurement Using Db40 Wavelets Decomposed

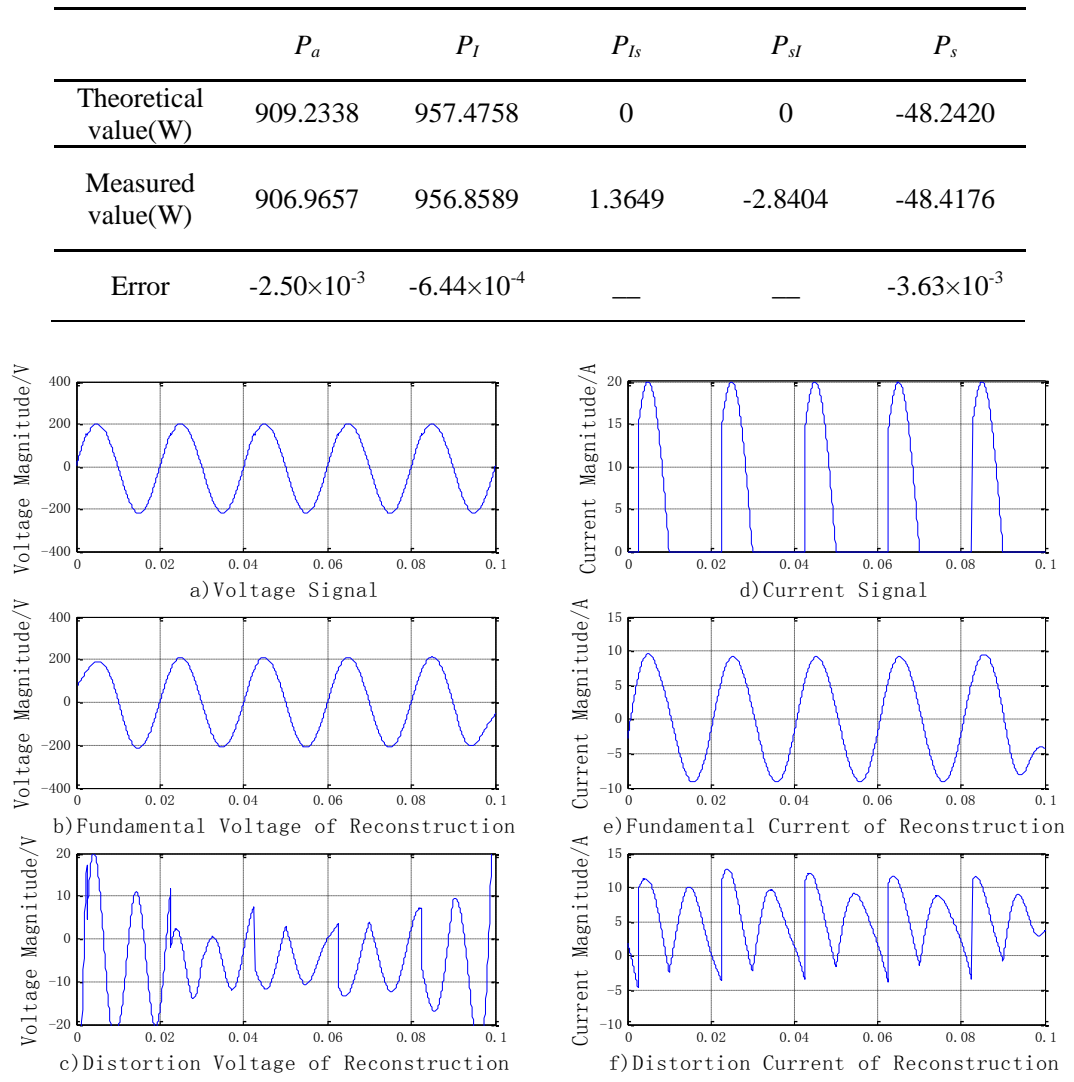

Figure 3. Distortion Wave and Fundamental Wave of Current and Voltage by Using Db40 and Sym11 Wavelets Decomposed

Table 2. Results of Power Measurement Using Db40 and Sym11 Wavelets Decomposed

\begin{tabular}{cccccc}
\hline & $P_{a}$ & $P_{I}$ & $P_{I s}$ & $P_{s I}$ & $P_{s}$ \\
\hline $\begin{array}{c}\text { Theoretical } \\
\text { value(W) }\end{array}$ & 909.2338 & 957.4758 & 0 & 0 & -48.2420 \\
\hline $\begin{array}{c}\text { Measured } \\
\text { value(W) }\end{array}$ & 909.8520 & 957.3005 & 1.3498 & -0.4583 & -48.3400 \\
\hline Error & $6.80 \times 10^{-4}$ & $-1.83 \times 10^{-4}$ & - & - & $-2.03 \times 10^{-3}$ \\
\hline
\end{tabular}

From the simulation result we know

1) From Figure 2 and Figure 3, comparing the fundamental component result of using Db40 wavelet basis decomposition with using sym11 wavelet basis decomposition, the result of using Sym11 wavelet basis is more tending the actual waveform. It shows that Sym11 wavelet basis more suiTable decomposition of fundamental component of distortion signals.

2) Comparing Table1 with Table 2, both Table1 and Table 2 reached the power accuracy level is $10^{-3}$, in measure total power $P_{a}$, the accuracy level reached $10^{-4}$ using multiple wavelets measurement method including the Db40 and Sym11, it shows that using the method of multiple wavelets can improve the measure accuracy of power.

3) The power measurement accuracy level of Table 2 more than Table 1. 


\subsection{Active Power Measurement of Electrical Arc Furnace}

It is calculated in theoretical that the active power theory value of electrical arc furnace consumption, fs $=12800 \mathrm{~Hz}$ is sampling frequency of simulation, $\mathrm{n}=5120$ is sampling points, and the signal is decomposed into 6 levels, Figure 4 and Table 3 are the result of using Db40 wavelet basis decomposition, Figure 5 and Table 4 are the distortion component result of using Db40 wavelet basis decomposition, and the fundamental component result of using Sym11 wavelet basis decomposition

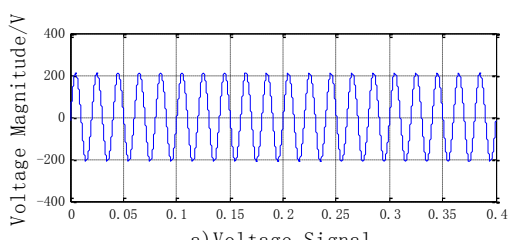

a) Voltage Signal
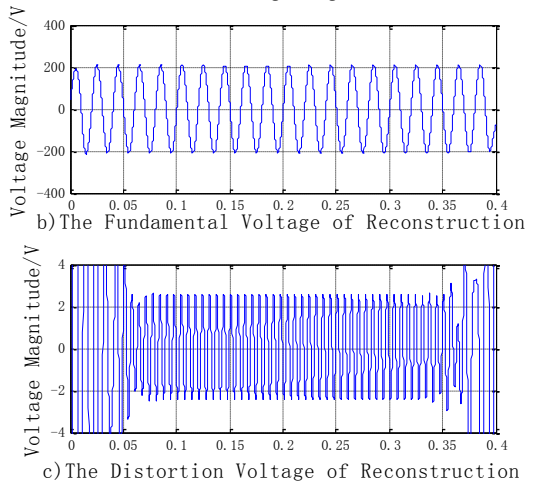

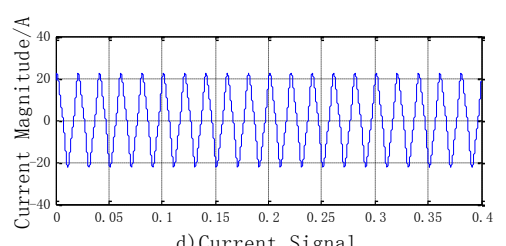

d) Current Signal
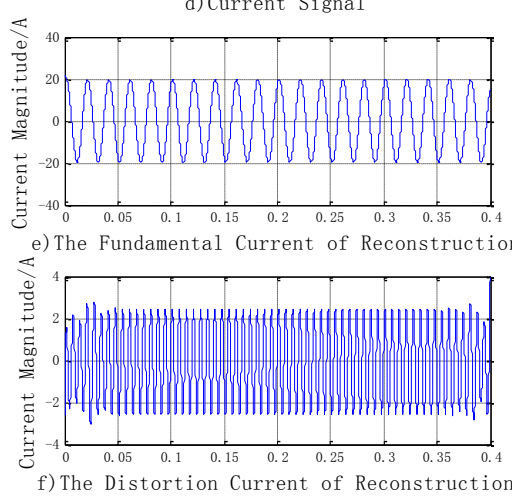

Figure 4. Distortion Wave and Fundamental Wave of Current and Voltage by Using Db40 Wavelets Decomposed

Table 3. Results of Power Measurement Using Db40 Wavelets Decomposed

\begin{tabular}{cccccc}
\hline & $P_{a}$ & $P_{I}$ & $P_{I s}$ & $P_{s I}$ & $P_{s}$ \\
\hline $\begin{array}{c}\text { Theoretical } \\
\text { value(W) }\end{array}$ & 896.8750 & 900.0000 & 0 & & -3.1250 \\
\hline $\begin{array}{c}\text { Measured } \\
\text { value(W) }\end{array}$ & 897.1930 & 900.2168 & 0.0941 & 0.0068 & -3.1248 \\
\hline Error & $3.55 \times 10^{-4}$ & $2.41 \times 10^{-4}$ & - & - & $1.67 \times 10^{-4}$ \\
\hline
\end{tabular}



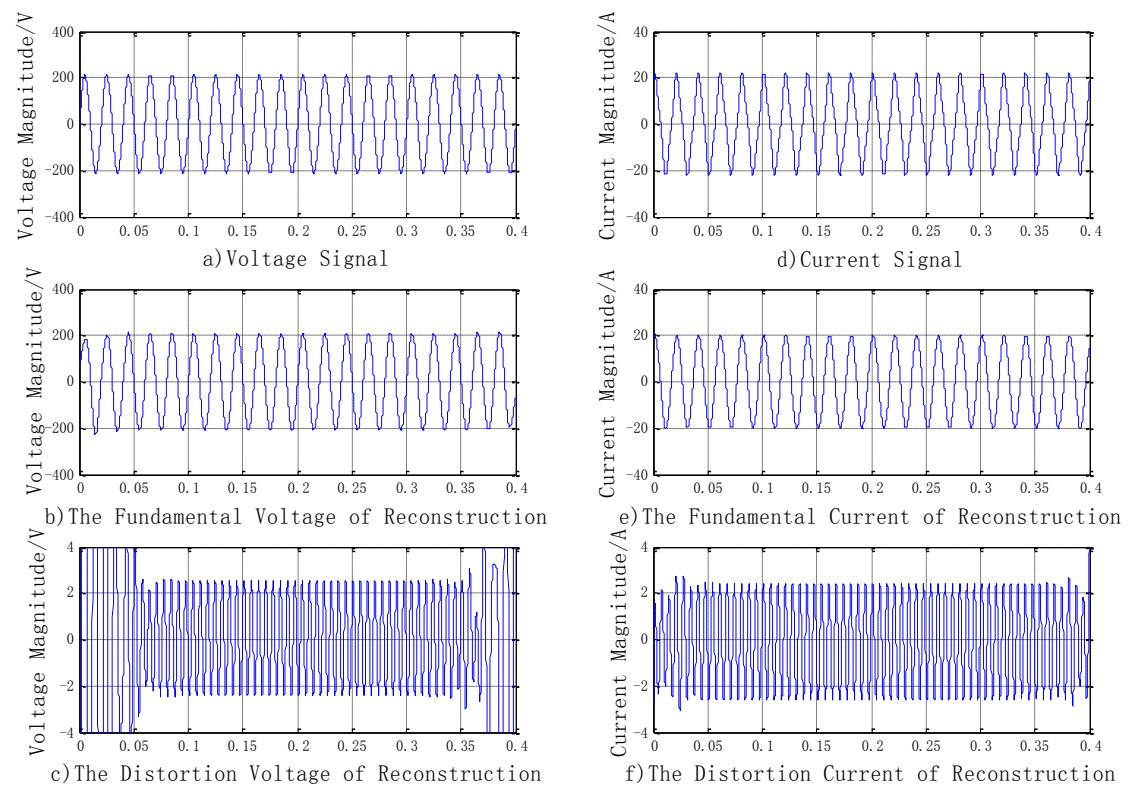
Figure 5. Distortion Wave and Fundamental Wave of Current and Voltage by
Using Db40 and Sym11 Wavelets Decomposed

Table 4. Results of Power Measurement using Db40 and Sym11 Wavelets Decomposed

\begin{tabular}{cccccc}
\hline & $P_{a}$ & $P_{I}$ & $P_{I s}$ & $P_{s I}$ & $P_{s}$ \\
\hline $\begin{array}{c}\text { Theoretical } \\
\text { value(W) }\end{array}$ & 896.8750 & 900.0000 & 0 & 0 & -3.1250 \\
\hline $\begin{array}{c}\text { Measured } \\
\text { value(W) }\end{array}$ & 896.9629 & 900.1032 & -0.0213 & 0.0069 & -3.1259 \\
\hline Error & $9.81 \times 10^{-5}$ & $1.15 \times 10^{-4}$ & - & - & $2.88 \times 10^{-4}$ \\
\hline
\end{tabular}

From the simulation result we know

1) From Figure 4 and Figure 5, comparing the fundamental component result of using Db40 wavelet basis decomposition with using sym11 wavelet basis decomposition, the result of using Sym11 wavelet basis is more tending the actual waveform. It shows that Sym11 wavelet basis more suitable decomposition of fundamental component of distortion signals.

2) Comparing Table 3 with Table 4 , both Table 3 and Table 4 reached the power accuracy level is $10^{-3}$, in measure total power $P_{a}$, the accuracy level reached $10^{-4}$ using multiple wavelets measurement method including the Db40 and Sym11, it shows that using the method of multiple wavelets can improve the measure accuracy of power.

\subsection{Simulation Error Analysis}

1) The marginal effect of Wavelet analysis would bring some error for wavelet transform.

2) Different signal have different optimal wavelet basis, so the selecting of wavelet basis would bring different error. 


\section{Conclusions}

A new multiple wavelets method of active power measurement was used measured semiconductor rectifier and harmonic model of electrical arc furnace, through simulation experiments, we have the conclusions:

1) It has different accuracy levels that using different wavelet basis for different frequency band of signal. The accuracy levels of using Db40 wavelet basis is more than using Sym11 wavelet basis on decomposition the distortion component of signal, and the accuracy levels of using Sym11 wavelet basis is more than using Db40 wavelet basis on decomposition the fundamental component of signal.

2) It is higher than wavelet transform that the accuracy levels of electrical energy measurement using multiple wavelets transform, and shows that the method of electrical energy measurement for different frequency band of signal using different wavelet basis is correct.

3) From the results of decomposition, the multiple wavelets analysis to load is more targeted, and more accuracy that decomposition frequency component from the signal.

\section{ACKNOWLEDGEMENTS}

Supported by Research Subject of Science and Technology of the Education Departme nt in HeiLongJiang Province (12531128)

\section{References}

[1] X. Ying and L. Baoshu, "The impact of nonlinear load on the voltage and current of the grid", Power System, vol. 26, no. 8, (2007), pp. 25-28.

[2] Y. Haiwen, Q. Ye, Z. Cheng, et al., "Research on harmonic current distribution of Beijing's rail traffic power supply system", //9th International Conference on Electronic Measurement \& Instruments Beijing China, The Chinese Institute of Electronics, (2009), pp. 235-238.

[3] Z. Junhua, W. Fushuan, Y. Aiming, et al., "Impacts of electric vehicles on power systems as well as the associated dispatching and control problem", Automation of Electric Power Systems, vol. 35, no. 14, (2011), pp. 2-10, 29.

[4] Y. Jinxiong, Z. Tao, L. Rong, et al., "Impacts of negative sequence current harmonics in traction power supply system for electrified railway on power system and compensation measures", Power System Technology, vol. 32, no. 9, (2008), pp. 61-64, 88.

[5] Y. Weijia, J. Ping and L. Feng, "Effects of harmonics on electric energy metering", East China Electric Power, vol. 34, no. 2, (2006), pp. 40-42.

[6] J. Weiyu and L. Guiying, "Research on the fundamental electric energy meter based on active filter and its application", Journal of Changsha University of Electric Power (Natural Science), vol. 18, no. 4, (2003), pp. 41-44.

[7] G. Yunpeng, T. Zhaosheng, W. He, et al., "Harmonic analysis based on Kaiser window interpolation FFT and its application", Proceedings of the CSEE, vol. 30, no. 4, (2010), pp. 43-48.

[8] Z. Xiaobing, "Electrical network power flow analysis and research on new measuring methods of electric energy on the condition of distorted signals", Harbin Harbin University of Science and Technology, (2007), pp. 5-31.

[9] L. Minggui, Y. Xianghong, Y. Yongbo, et al., "Intelligent identification of pile defect based on Sym wavelet and BP neural network", Chinese Journal of Rock Mechanics and Engineering, vol. 26, no. 1, (2007), pp. 3484-3488. 
International Journal of Hybrid Information Technology Vol.8, No.6 (2015) 